\title{
Cosmology and Neutrino Physics
}

\author{
Raul Jimenez ${ }^{* \dagger}$ \\ ICCUB, University of Barcelona (UB-IEEC), Marti i Franques 1, Barcelona, 08028, Spain. \\ E-mail: raul.jimenez@icc.ub.edu
}

\begin{abstract}
I review the current status of the cosmological standard model (LCDM) with a special focus on neutrino cosmology. In particular, I describe how the standard model of cosmology has, so far, passed all tests and thus is able to explain all current observations of the sky. Within this model, it is possible to infer properties of neutrinos. In particular, I show how current cosmological surveys constraint, robustly, the sum of neutrino masses $<0.13 \mathrm{eV}$ and that current cosmological data already favour strongly, in a Bayesian sense, the normal ordering of the neutrino masses. In the next five years, future cosmological surveys should be able to measure robustly the sum of neutrino masses and, further in the future, the individual masses of neutrinos.
\end{abstract}

XVII International Workshop on Neutrino Telescopes

13-17 March 2017

Venezia, Italy

\footnotetext{
*Speaker.

${ }^{\dagger}$ I thank my long term collaborators in neutrino cosmology Carlos Pena-Garay and Licia Verde
} 


\section{Introduction}

In the past few years, there has been an amazing progress in cosmology. An accurate cosmic microwave background $(\mathrm{CMB})$ angular power spectrum, both in temperature and polarization has been measured by WMAP [1] and Planck [2]. The expansion history of the Universe has been mapped in several ways: with measurements of the Baryon Acoustic Oscillation (BAO) scale by the Baryon Oscillation Spectroscopic Survey (BOSS) of the Sloan Digital Sky Survey (SDSS) [3] and others $[4,5]$; by the luminosity distance relation as given by Type 1A supernova data e.g., [6]; via the direct measurement of the Hubble parameter with cosmic chronometers [7, 8].

Finally, large scale structure (LSS) has been probed by a variety of surveys (galaxies e.g., $[9,10,11,12]$, weak lensing e.g., $[13,14,15,16,17]$, Ly $\alpha$ [18]) with increased sensitivity to the scale and redshift dependences of the matter power spectrum, thanks also to redshift space distortion measurements e.g., $[19,20]$.

All this wealth of cosmological data show a consistent $\Lambda$ CDM model with improved precision on parameters and better control of systematics. If included as a parameter in the model, total neutrino mass bounds have significantly improved in a variety of analysis, yielding an upper bound slightly higher than $100 \mathrm{meV}[18,21]$. Massive neutrinos free stream out of potential wells, erasing fluctuations and thus suppressing power on small scales e.g., [22, 23]; the measured small-scale power is consistent with the standard (massless neutrino) $\Lambda \mathrm{CDM}$ model and inconsistent with large neutrino masses. These bounds are very close to the limit that separates inverted and normal ordering and is within a factor of two of the lower limit of the sum of neutrino masses set by oscillations e.g., [24]. Near-future progress in this measurement has an important impact on a crucial question in neutrino physics [25]: is neutrino its own anti-particle? The almost century-old question can be resolved if a neutrinoless double beta decay is observed [26, 27]. If light neutrinos are the main source of this decay, the measurement of the neutrino mass, provides an estimate of the half-life and therefore the size and level of background of experiments required to prove it.

Massive neutrinos affect cosmological observations in a variety of different ways. For example, the combination of CMB and BAO data constrain the total neutrino mass $\Sigma<0.23 \mathrm{eV}$ at the $95 \%$ confidence level [2]. Neutrinos with mass $\lesssim 1 \mathrm{eV}$ become non-relativistic after the epoch of recombination probed by the $\mathrm{CMB}$, thus massive neutrinos alter matter-radiation equality for a fixed physical matter density $\Omega_{m} h^{2}$. After neutrinos become non-relativistic, their free streaming damps the small-scale power and modifies the shape of the matter power spectrum below the freestreaming length. Combining large-scale structure and CMB data, at present the sum of masses is constrained to be $\Sigma \lesssim 0.13 \mathrm{eV}$ [21, 18]. Forthcoming large-scale structure data promise to determine the small-scale $(0.1 \lesssim k \lesssim 1 \mathrm{~h} / \mathrm{Mpc})$ matter power spectrum exquisitely well and to yield errors on $\Sigma$ well below $0.1 \mathrm{eV}$ (e.g., [28, 29]).

Cosmological surveys provide crucial information on the absolute masses of neutrinos, information that is not accessible even to future laboratory experiments [32, 33, 34]. Recent analyses have already established a robust upper limit on the sum of neutrino masses of $\Sigma<0.13 \mathrm{eV}$ [31] and $\Sigma<0.12$ [18] at 95\% confidence (throughout this work we shall denote $\Sigma m_{v}$ as $\Sigma$ for brevity). This limit is tantalisingly close to the lower limit for the sum of the masses in the inverted hierarchy $\Sigma=0.0982 \pm 0.0010 \mathrm{eV}$ (68\% C.L.) [30]. Because the volume covered by cosmological surveys keeps increasing, their statistical errors shrink, soon leading to either (a) a measure the total neu- 
trino mass or (b) the exclusion of the inverted hierarchy, leaving the normal one as the only viable option. It is therefore possible to determine the neutrino hierarchy, even in the absence of a measurement of the total mass e.g., Ref. [28, 29, 35]. Future surveys may even yield a measurement of the mass differences between the lightest and heaviest eigenstates [36]. This measurement would also provide a powerful consistency check: the measured mass splitting from cosmology should be consistent with the squared mass splitting from oscillations.

Neutrino oscillation data have measured the neutrino squared mass differences, which are hierarchical. Given the smallness of neutrino masses and the hierarchy in mass splittings, we can characterize the impact of neutrino masses on cosmological observables and in particular on the matter power spectrum by two parameters: the total mass $\Sigma$ and the ratio of the largest mass splitting to the total mass, $\Delta$; while one can safely neglect the impact of the solar mass splitting in cosmology. In this excellent approximation, two masses characterize the neutrino mass spectrum, the lightest one, $m$, and the heaviest one, $M$.

Relevant cosmological constraints on neutrino masses (i.e., coming from measurements of the small scales power suppression) have to be matched with careful verification of systematic effects or alternative explanations of the data. Building on the work of [25], we show that cosmological data contain information on the neutrino masses additional to the power suppression, statistically independent from it, and in a form that can hardly be mimicked by other effects. Such footprint is fully described by the measured power suppression (an astrophysical measurement) and atmospheric neutrinos mass splitting (a neutrino oscillation experiment measurement). Driven by the current strong limits in the total mass, we demonstrate that if the total mass is measured from forthcoming cosmological data then there is sensitivity to detect the footprint and measure from it the expected large mass splitting. The agreement of the mass splitting inferred from cosmology and the large neutrino mass splitting measured by oscillation experiments should prove as convincing evidence of the discovery of relic neutrinos from cosmological surveys.

\section{Cosmology, oscillations, and statistics}

In this section we provide a brief review of the three disciplines that are central to current research in neutrino cosmology.

\subsection{Cosmology: observational constraints and degeneracies}

Massive neutrinos influence both the expansion history and growth of structure in the Universe. Neutrinos of mass $\lesssim 1 \mathrm{eV}$ become non-relativistic after the epoch of recombination probed by the Cosmic Microwave Background (CMB), and this mechanism allows massive neutrinos to alter the relationship connecting the physical matter density $\Omega_{m} h^{2}$ to the redshift of matter-radiation equality. The neutrinos' radiation-like behaviour at early times modulates the peak positions in the CMB angular power spectrum. However this effect is somewhat degenerate with other cosmological parameters. Neutrinos are also responsible for dampening the matter power spectrum below their free-streaming scale; see e.g., [23] for a review. This effect, which amounts to a scale dependent power suppression, is straightforward to compute in linear theory [37] and in the non-linear regime via numerical simulations e.g., $[38,39,40]$. 
Due to these mechanisms, cosmological observables are predominantly sensitive to the sum of neutrino masses $\Sigma$ e.g., [41, 42, 43, 23, 44, 45, 46]. While the effect of neutrino mass on the $\mathrm{CMB}$ is related to the physical density of neutrinos, and therefore the mass difference between eigenstates can be neglected, individual neutrino masses, however, can have an effect on the largescale shape of the matter power spectrum. Neutrinos of different masses have different transition redshifts from relativistic to non-relativistic behaviour, and their mass splitting changes the details of the radiation-domination to matter-domination regime. A precise measurement of the matter power spectrum shape can give information on both the sum of the masses and individual masses (and thus the hierarchy), even if the second effect is much smaller than the first [36].

There are only two independent neutrino mass squared differences. In this convention $m_{1}<m_{2}$ and $m_{1}, m_{2}$ refer to the smaller square mass difference. Thus there are two possible hierarchies only. In what is known as the normal mass hierarchy, $m_{1}, m_{2}$, and $m_{3}$ are defined in ascending order, $m_{1} \leq m_{2} \leq m_{3}$. For the case of the inverted hierarchy, the sequence becomes $m_{3} \leq m_{1} \leq m_{2}$. In other words the hierarchy does not strictly corresponds to whether we have two small masses and one large or vice versa, this arises only once the results of the measurements of atmospheric and solar oscillations square mass splitting are in hand; the hierarchy is given by the sign of the square mass splitting involving $m_{3}$.

We define the relation between the neutrino masses $m$ and $M$ and the parameters $\Sigma$ and $\Delta$ as

$$
\begin{array}{ccc}
\mathrm{NH}: & \Sigma=2 m+M & \Delta=(M-m) / \Sigma \\
\mathrm{IH}: & \Sigma=m+2 M & \Delta=(m-M) / \Sigma .
\end{array}
$$

Significant progress has been made by cosmological surveys to constrain the total mass of neutrinos. For example, the latest Planck satellite results show that CMB data alone constrain $\Sigma<0.59$ $\mathrm{eV}$ (95\% C.L.) [2], where the upper limit is stronger than $\sim 1 \mathrm{eV}$ because of the extra constraining power offered by secondary effects, mostly lensing. The inclusion of late-time information about the cosmic expansion history, such as that provided by measurements of the Baryon Acoustic Oscillations (BAO), further breaks cosmological parameters degeneracies yielding a limit $\Sigma<0.17$ eV (95\% C.L.).

Including information about the shape of the matter power spectrum further tightens these constraints, even when BAO information is not used. In Ref. [31] the authors have carefully examined the impact of using different galaxy populations and tracers to estimate the power spectrum of the underlying dark matter and concluded that $\Sigma<0.13 \mathrm{eV}$ at $95 \%$ confidence. This constraint tightens to $\Sigma<0.11 \mathrm{eV}$ if also the latest measurement of the Hubble constant [47] are included. Ref. [31] result is consistent with the previous constraint from a different estimate of the power spectrum of dark matter using the Lyman- $\alpha$ forest [18], $\Sigma<0.12 \mathrm{eV}$ (see also [48, 49]).

All the above constraints assume a standard cosmological model, where the spatial geometry is flat, dark matter (excluding the neutrino component) is cold, the dark energy is a cosmological constant, general relativity is the correct description of gravity on large, cosmological scales, the initial conditions of the Universe are given by a process like inflation and thus the primordial power spectrum is a nearly scale invariant power law. In addition, it is implicitly assumed that modelling of real world effects such as tracer's bias, non-linearities, etc. is sufficiently accurate as not to induce systematic errors that are larger than the reported ones. Relaxing any of these assumptions would weaken the constraints significantly e.g., [50, 51, 52]. 
Detecting the effect of neutrino masses on cosmological structure and resolving the neutrino mass scale is well within the reach of up-coming cosmological surveys (e.g., [53, 28, 29] and references therein).

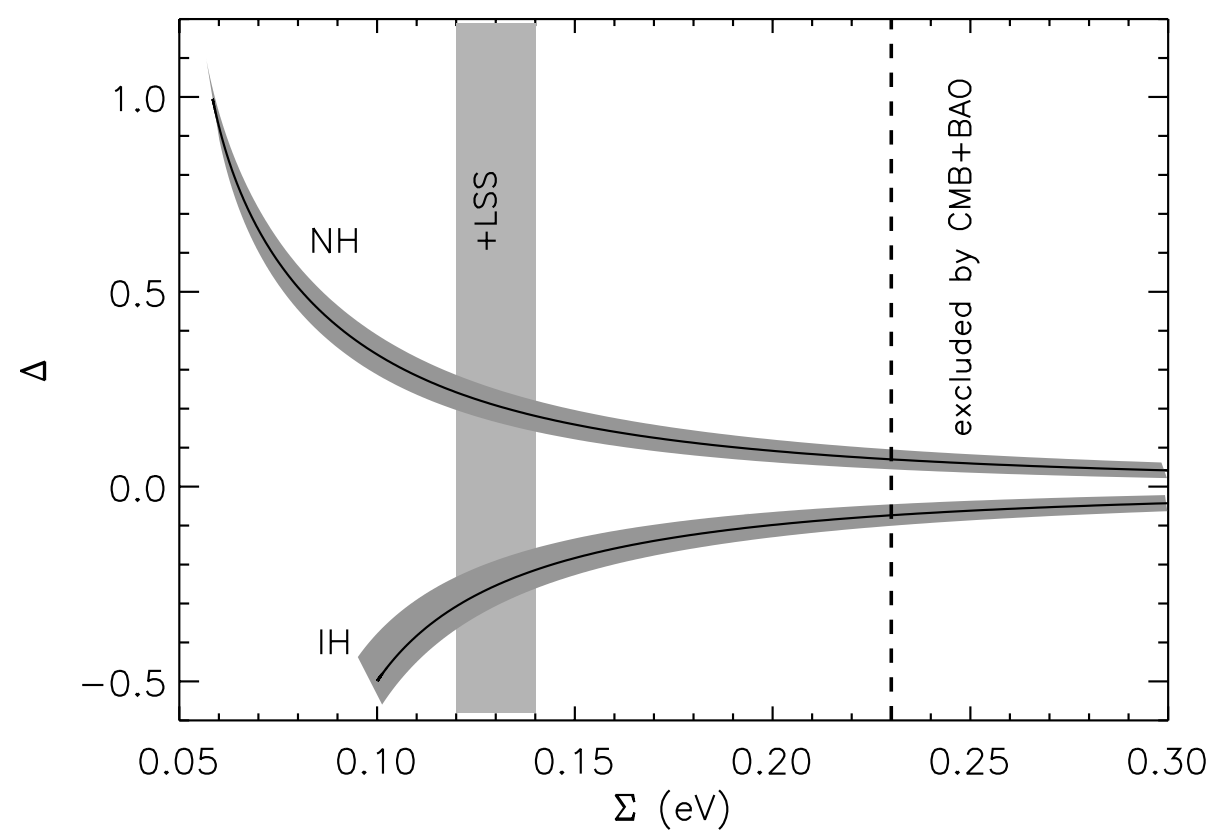

Figure 1: Constraints from neutrino oscillations (shaded regions) and from cosmology in the $\Sigma-\Delta$ plane. In this parameterization the sign of $\Delta$ specifies the hierarchy.

\subsection{Neutrino Experiments}

Solar, atmospheric, reactor and accelerator neutrino experiments have observed neutrino flavor conversion driven by neutrino masses. The experiments that mostly contribute to a precise measurement of the neutrino masses are sensitive to the relative phases acquired by relativistic neutrinos on their way from the production zone to the detector. Therefore the neutrino oscillation observables are sensitive to mass splittings, specifically to differences in the square of their masses. Matter effects allow the sign of the mass splittings to be distinguished, a goal which has been achieved in the small mass squared splitting but not the large one. The degeneracy in this second case distinguishes the two hierarchy mass orderings, normal and inverted. Identifying the neutrino mass hierarchy is a key objective for future neutrino oscillation experiments, in addition to a determination of the complex phase involved in flavor conversion.

The absolute neutrino mass is not accessible via flavor conversion experiments, but it can be probed using beta decay. Experiments require sufficient mass and very good energy resolution in order to measure the beta spectrum close to the endpoint, where the neutrino energy is the neutrino mass. The experiments measure the effective mass of the electron, which is the combination of 
neutrino masses weighted by their content in the electron flavor. The present bound derived from the electron mass is $2.3 \mathrm{eV}(\Sigma<6.9 \mathrm{eV})$ at $90 \%$ confidence. The KATRIN experiment is expected to significantly improve upon this bound, reaching approximately $0.2 \mathrm{eV}(\Sigma<0.6 \mathrm{eV})$ at $90 \%$ [32]. Meanwhile new proposals [33] like Project 8 [34] aim for yet greater sensitivity.

\subsection{Bayesian Model Selection}

It is now the standard to employ Bayesian model selection in order to quantify the manner in which the combination of existing data, from both cosmology and neutrino experiments, should influence our belief in the normal or inverted hierarchy. Each hypothesis possesses the same number of free parameters, facilitating a fair comparison between the two. Furthermore their low dimensionality (three masses) permits an exact evaluation of the Bayesian evidence, a process which is often highly impractical for more complex models.

The Bayesian evidence $p(D \mid \mathscr{H})$ (the probability of the data $D$ given the hypothesis $\mathscr{H}$ ) is largely ignored during conventional analyses of cosmological parameter estimation, as it merely takes the form of a normalising constant in Bayes theorem

$$
p(\alpha \mid D, \mathscr{H})=\frac{p(D \mid \alpha, \mathscr{H}) p(\alpha \mid \mathscr{H})}{p(D \mid \mathscr{H})} .
$$

where $p(D \mid \alpha, \mathscr{H})$ is the likelihood, $\alpha$ denote the parameters of the model, and $p(\alpha \mid \mathscr{H})$ the prior. However, when it comes to determining our preference for one hypothesis over another, the evidence plays a central role. In order to evaluate the evidence it may be expressed in terms of the likelihood and the prior probability $\pi=p(\alpha \mid \mathscr{H})$ as follows

$$
p(D \mid \mathscr{H})=\int p(D \mid \alpha, \mathscr{H}) p(\alpha \mid \mathscr{H}) d \alpha .
$$

The ratio of evidences for two competing hypotheses, $\mathscr{H}_{1}$ and $\mathscr{H}_{2}$, is referred to as the Bayes factor, and this represents the key quantity in Bayesian model selection,

$$
K=\frac{p\left(D \mid \mathscr{H}_{1}\right)}{p\left(D \mid \mathscr{H}_{2}\right)} .
$$

Mechanisms which lead us to prefer one model over another can be crudely decomposed into two contributing factors: the peak and the breadth of the integrand given in (2.4). These two quantities are known as the maximum likelihood value, and the so-called Occam factor. If one hypothesis is capable of fitting the data better than another, then it will yield a greater value of $p(D \mid \alpha, \mathscr{H})$, and this modifies our belief accordingly. Of greater significance is the second contribution to the evidence, which relates to the predictivity of the model. This concept was succinctly summarised by MacKay [54] as follows:

"The Occam factor is equal to [...] the factor by which $\mathscr{H}$ 's hypothesis space collapses when the data arrive."

In other words, the Occam factor penalises complex models; these can be models having many parameters or, as in this case when the two models we compare have the same number of parameters, the complexity of the predictions that the model makes. In this case the Occam factor penalises models that have to be fine tuned to fit the data. Thus the Bayes factor balances the quality 
of the fit versus the model complexity, as such it rewards highly predictive models and penalises models with "wasted" parameter space (i.e., it favours models which offer a good fit over large part of the prior volume). Inevitably the evidence calculation thus depends on the choice of prior and prior ranges. Our prior choice will be discussed below. Therefore if a much greater fraction of one hierarchy's three-dimensional parameter volume is excluded by the data, we should favour the other hierarchy (see Fig. 2).

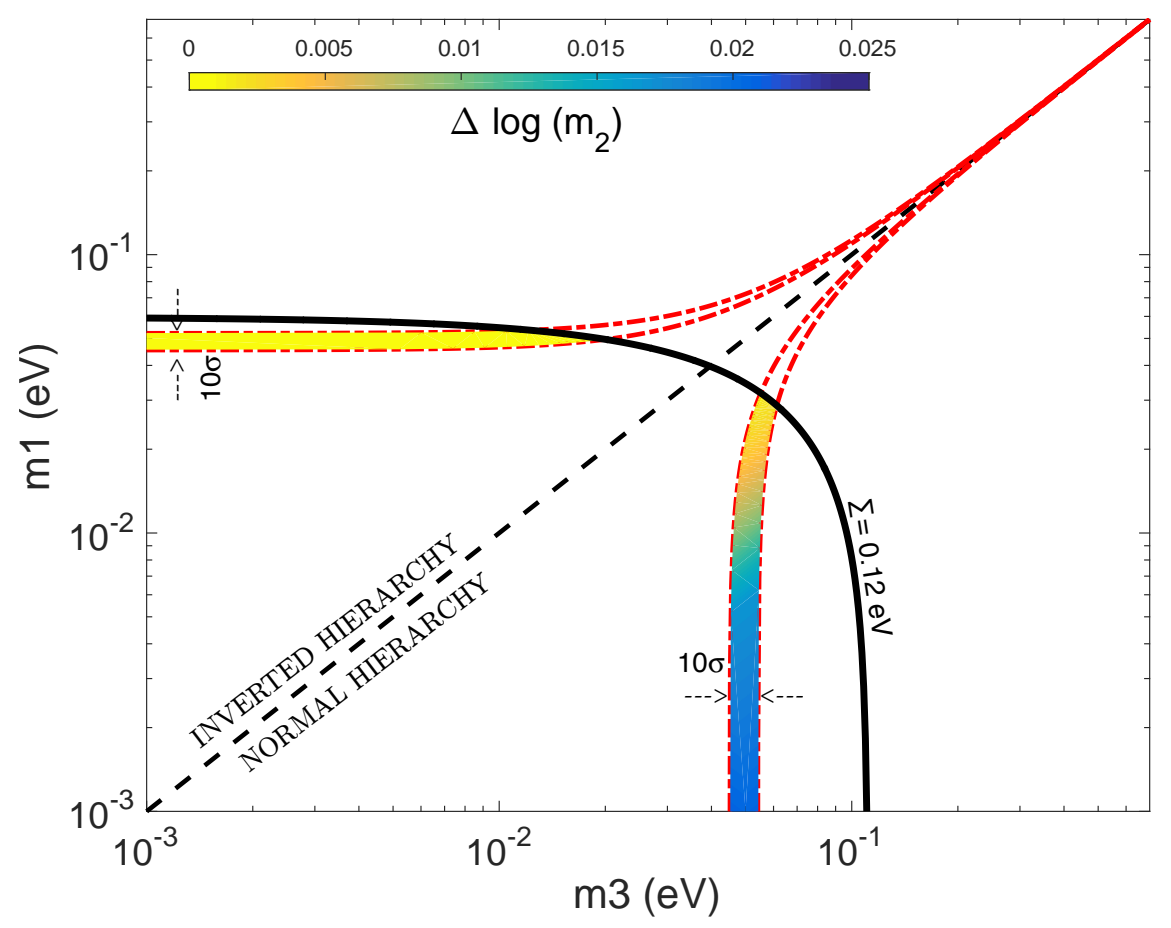

Figure 2: A visualisation of the heavily reduced parameter space available in the case of the inverted neutrino mass hierarchy, relative to the normal case. The red dash-dot contours illustrate constraints on the mass splittings, as imposed by neutrino oscillation experiments (broadened to show $10 \sigma$ uncertainties for visualisation purposes). The solid black line corresponds to the combination of a cosmological upper bound on the sum of the neutrino masses $\Sigma<0.12 \mathrm{eV}$ with the measurement of $\Delta m_{12}^{2}$. The diagonal dashed line demarcates the two hierarchies. The colouring of the shaded areas represents the amount of parameter space available in the third dimension, $\Delta\left(\log m_{2}\right)$.

\subsection{Interpreting results from upcoming surveys}

Fig. 3 serves as guidance for the interpretation of forthcoming results from cosmology. In order to facilitate a comparison with conventional cosmological analyses, we present our results in terms of the $95 \%$ confidence bounds which would be achieved when imposing a uniform prior on $\Sigma$, and without utilising external information from neutrino oscillation experiments. The solid line corresponds to the family of Gaussian likelihoods $p\left(D_{\Sigma} \mid \Sigma\right)$, of varying standard deviation but each with maxima located at $0 \mathrm{eV}$, as motivated by the findings of recent analyses. Meanwhile the dashed line demonstrates the impact of shifting the maximum likelihood value to $0.05 \mathrm{eV}$. These lines illustrate the degree of belief we would reach in the normal neutrino hierarchy, when this hypothetical cosmological data is combined with the squared mass splittings. What if these 


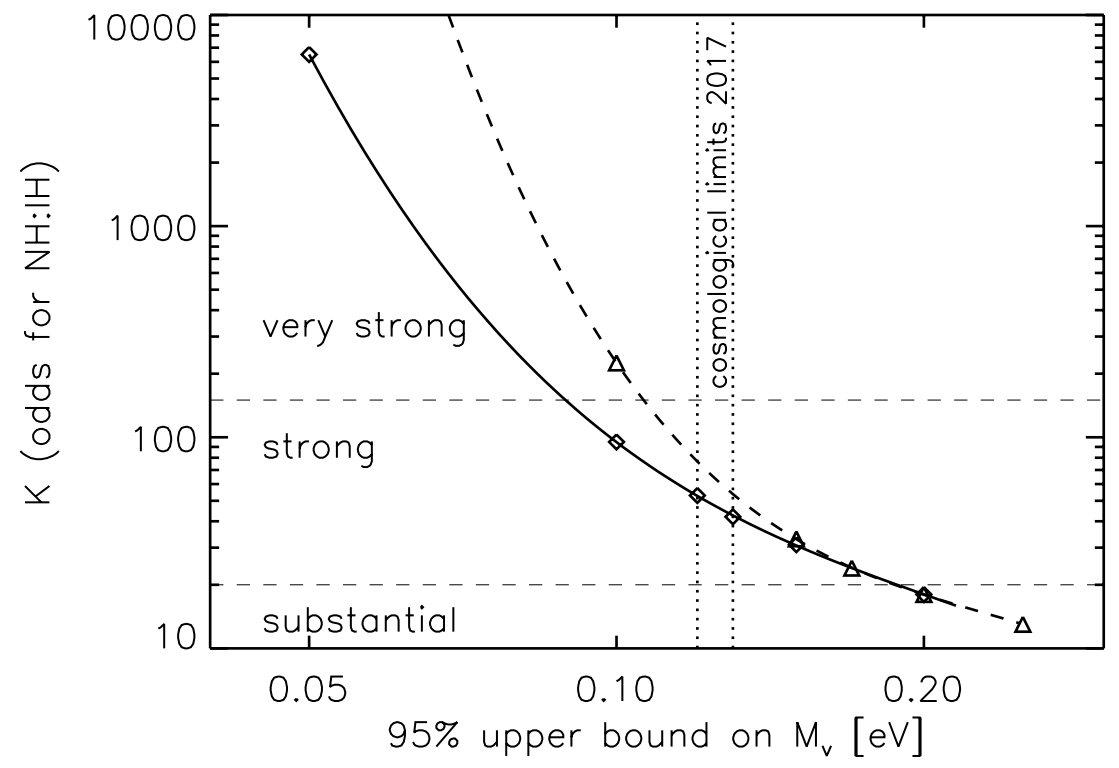

Figure 3: Odds as a function of cosmological upper limits on the sum of neutrino masses $\Sigma$. The solid line corresponds to the (actual) case where the maximum of the $\Sigma$ distribution is indistinguishable from zero. The dashed line correspond to a case where maximum of the $\Sigma$ distribution is at $0.05 \mathrm{eV}$. Also indicated (vertical dotted lines) are the current limits from CMB and clustering of galaxies [31] or Lyman $\alpha$ forest [18]. Jeffreys' interpretation of the Bayes factor values are also reported.

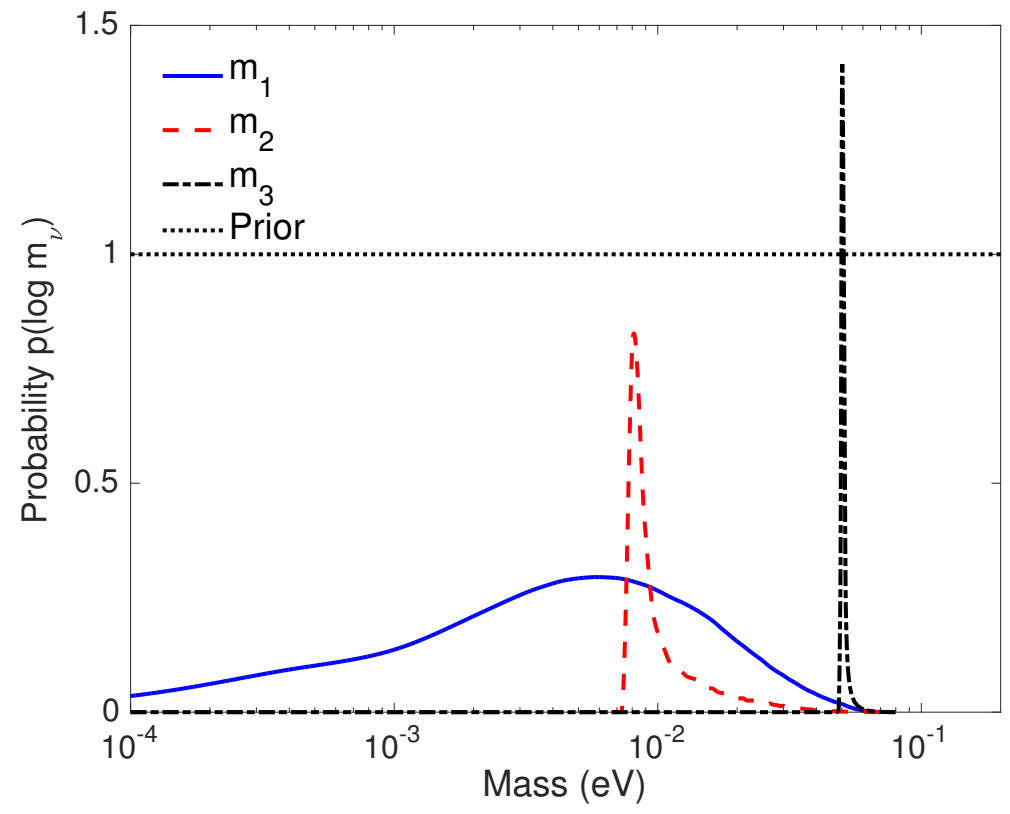

Figure 4: The posterior distribution on the three individual masses, for the case of the normal hierarchy. To reach these constraints we utilise $\Sigma<0.12 \mathrm{eV}$ cosmological bound. The amplitudes of the distributions are rescaled for clarity. 
hypothetical future measurements provide instead a likelihood for $\Sigma$ which is centred at a value close to the current $95 \%$ upper limit? In this case, if this value is e.g., $0.1 \mathrm{eV}$, the preference for normal hierarchy is much weaker but still a preference remains. For example for 1- $\sigma$ errors of $\{0.005,0.01,0.025,0.05\} \mathrm{eV}$ corresponding to $95 \%$ upper limits of $\{0.11,0.12,0.15,0.2\} \mathrm{eV}$, one would obtain odds of $\{3.7,5.3,11,12\}$ to 1 in favour of $\mathrm{NH}$.

\subsection{Constraints on the individual masses}

Figure 4 presents the posterior distributions on the three individual neutrino masses. In light of our findings in the previous section, here we only consider the normal hierarchy's contribution to the posterior. Unsurprisingly the lightest mass is the least well constrained, with a mild preference that it is not very far in log space from the other two masses. Meanwhile the two heavier masses, $m_{2}$ and $m_{3}$, possess sharper but highly asymmetric posteriors. From these posterior distributions we may derive medians and credible intervals for each mass eigenstate, yielding $m_{1}=3.80_{-3.73}^{+26.2} \mathrm{meV} ; m_{2}=8.8_{-1.2}^{+18} \mathrm{meV} ; m_{3}=50.4_{-1.2}^{+5.8} \mathrm{meV}(95 \% \mathrm{CL})$.

Finally, the dotted horizontal line illustrates our belief in each of the masses before the data arrives. We stress that the difference between the prior and the posterior distribution is unequivocally driven by the data.

\section{Implications in neutrino physics}

The determination of the nature of neutrinos, whether Majorana or Dirac fermions, is a major long-term unsolved problem in particle physics, with double beta decay experiments being the most sensitive probes to solve this riddle. These experiments could prove the existence of a rare double beta decay of nuclei without neutrino emission, a decay which is mediated by the internal exchange of the light Majorana neutrinos (other beyond the standard model physics may also contribute to the decay). In the minimal case, the expected half-lives of neutrino-less double beta decays of nuclei depend on nuclear physics dynamics and on the neutrino mass scale and hierarchy. In fact, the neutrino mass hierarchy plays a key role in identifying the largest half-lives to be explored [?]. While it is true that, independently of the neutrino mass hierarchy, the discovery could be close to the upper bounds, for example $10^{27} \mathrm{yr}$ for Xe [55], the strong evidence for the normal neutrino mass hierarchy favours experimental techniques with potential to reach multi-ton active mass detectors and meet the requirements of very low background with, for example, topological signatures[56, 57].

Neutrino mass hierarchy is among the physics goals of several proposed neutrino detectors [58, 59, 60, 61, 62]. Experiments with observables more sensitive to normal mass hierarchy are much more likely to yield a successful outcome.

Is there a means by which our conclusion can be evaded? One can always resort to invoking unknown systematics large enough to invalidate the observational data, or hoping that the LCDM cosmological model is wildly inaccurate, but these appear to be highly speculative escape routes.

\section{References}

[1] G. Hinshaw et al. [WMAP Collaboration], Astrophys. J. Suppl. 208, 19 (2013) 
[2] P. A. R. Ade et al. [Planck Collaboration],

[3] L. Anderson et al. [BOSS Collaboration], Mon. Not. Roy. Astron. Soc. 441, no. 1, 24 (2014)

[4] C. Blake et al., Mon. Not. Roy. Astron. Soc. 418, 1707 (2011)

[5] F. Beutler et al., Mon. Not. Roy. Astron. Soc. 416, 3017 (2011)

[6] M. Betoule et al. [SDSS Collaboration], Astron. Astrophys. 568 (2014) A22

[7] M. Moresco et al., JCAP 1208 (2012) 006

[8] Moresco, M., Pozzetti, L., Cimatti, A., et al. 2016, JCAP, 5, 014

[9] D. Parkinson et al., Phys. Rev. D 86 (2012) 103518

[10] S. Riemer-S£rensen, D. Parkinson and T. M. Davis, Phys. Rev. D 89 (2014) 103505

[11] B. A. Reid et al., Mon. Not. Roy. Astron. Soc. 404 (2010) 60

[12] S. Alam et al. [SDSS-III Collaboration], Astrophys. J. Suppl. 219 (2015) 1, 12

[13] Jee, M. J., Tyson, J. A., Hilbert, S., et al. 2016, ApJ, 824, 77

[14] L. Van Waerbeke et al., Mon. Not. Roy. Astron. Soc. 433 (2013) 3373

[15] M. Kilbinger et al., Mon. Not. Roy. Astron. Soc. 430 (2013) 2200

[16] T. D. Kitching et al. [CFHTLenS Collaboration], Mon. Not. Roy. Astron. Soc. 442 (2014) 2, 1326

[17] T. Abbott et al. [DES Collaboration], arXiv:1507.05552 [astro-ph.CO].

[18] N. Palanque-Delabrouille et al., JCAP 1511, no. 11, 011 (2015)

[19] L. Samushia et al., Mon. Not. Roy. Astron. Soc. 439 (2014) 4, 3504

[20] F. Beutler et al. [BOSS Collaboration], Mon. Not. Roy. Astron. Soc. 443 (2014) 2, 1065

[21] Cuesta, A. J., Niro, V., \& Verde, L. 2016, Physics of the Dark Universe, 13, 77

[22] W. Hu, D. J. Eisenstein and M. Tegmark, Phys. Rev. Lett. 80 (1998) 5255

[23] J. Lesgourgues and S. Pastor, Phys. Rept. 429 (2006) 307

[24] Gonzalez-Garcia, M. C., Maltoni, M., \& Schwetz, T. 2016, Nuclear Physics B, 908, 199

[25] R. Jimenez, T. Kitching, C. Pena-Garay and L. Verde, JCAP 1005 (2010) 035

[26] W. H. Furry, Phys. Rev. 56, 1184 (1939).

[27] E. Majorana, Nuovo Cim. 14, 171 (1937).

[28] C. Carbone, L. Verde, Y. Wang and A. Cimatti, JCAP 1103 (2011) 030

[29] B. Audren, J. Lesgourgues, S. Bird, M. G. Haehnelt and M. Viel, JCAP 1301 (2013) 026

[30] M. C. Gonzalez-Garcia, M. Maltoni, and T. Schwetz, JHEP 11, 052 (2014), 1409.5439.

[31] A. J. Cuesta, V. Niro, and L. Verde, Physics of the Dark Universe 13, 77 (2016), 1511.05983.

[32] A. Osipowicz et al. (KATRIN) (2001), hep-ex/0109033.

[33] B. Monreal and J. A. Formaggio, Phys. Rev. D80, 051301 (2009), 0904.2860.

[34] P. J. Doe et al. (Project 8), in Proceedings, Community Summer Study 2013: Snowmass on the Mississippi (CSS2013): Minneapolis, MN, USA, July 29-August 6, 2013 (2013), 1309.7093 , URL https://inspirehep.net/record/1255861/files/arXiv:1309.7093.pdf. 
[35] J. Hamann, S. Hannestad, and Y. Y. Y. Wong, JCAP 1211, 052 (2012), 1209.1043.

[36] R. Jimenez, T. Kitching, C. Pena-Garay, and L. Verde, JCAP 1005, 035 (2010), 1003.5918.

[37] J. R. Bond, G. Efstathiou, and J. Silk, Phys. Rev. Lett. 45, 1980 (1980).

[38] J. Brandbyge and S. Hannestad, JCAP 1001, 021 (2010), 0908.1969.

[39] C. Wagner, L. Verde, and R. Jimenez, Astrophys. J. 752, L31 (2012), 1203 . 5342.

[40] S. Bird, M. Viel, and M. G. Haehnelt, Mon. Not. Roy. Astron. Soc. 420, 2551 (2012), 1109.4416.

[41] W. Hu, D. J. Eisenstein, and M. Tegmark, Physical Review Letters 80, 5255 (1998), astro-ph/9712057.

[42] J. Lesgourgues, S. Pastor, and L. Perotto, PRD 70(4), 045016 (2004), hep-ph/ 0403296.

[43] S. Wang, Z. Haiman, W. Hu, J. Khoury, and M. May, Physical Review Letters 95(1), 011302 (2005), astro-ph/0505390.

[44] M. Takada, E. Komatsu, and T. Futamase, PRD 73(8), 083520 (2006), a st ro-ph/ 0512374.

[45] S. Hannestad and Y. Y. Y. Wong, JCAP 7, 004 (2007), a stro-ph/ 0703031.

[46] T. D. Kitching, A. F. Heavens, L. Verde, P. Serra, and A. Melchiorri, PRD 77(10), 103008 (2008), 0801.4565.

[47] A. G. Riess, L. M. Macri, S. L. Hoffmann, D. Scolnic, S. Casertano, A. V. Filippenko, B. E. Tucker, M. J. Reid, D. O. Jones, J. M. Silverman, R. Chornock, P. Challis, et al., ApJ 826, 56 (2016), 1604.01424.

[48] E. Giusarma, M. Gerbino, O. Mena, S. Vagnozzi, S. Ho, and K. Freese, PRD 94(8), 083522 (2016), 1605.04320 .

[49] E. Di Valentino, E. Giusarma, O. Mena, A. Melchiorri, and J. Silk, PRD 93(8), 083527 (2016), 1511.00975.

[50] R. de Putter, E. V. Linder, and A. Mishra, Phys. Rev. D89(10), 103502 (2014), 1401.7022.

[51] T. Namikawa, S. Saito, and A. Taruya, JCAP 1012, 027 (2010), 1009.3204.

[52] M. Baldi, F. Villaescusa-Navarro, M. Viel, E. Puchwein, V. Springel, and L. Moscardini, MNRAS 440, 75 (2014), 1311.2588.

[53] R. Jimenez, T. Kitching, C. Peña-Garay, and L. Verde, JCAP 5, 035 (2010), 1003.5918.

[54] D. J. MacKay, Information theory, inference and learning algorithms (Cambridge university press, 2003).

[55] A. Gando et al. (KamLAND-Zen), Phys. Rev. Lett. 117(8), 082503 (2016), [Addendum: Phys. Rev. Lett.117,no.10,109903(2016)], 1605.02889.

[56] F. Granena et al. (NEXT) (2009), 0907.4054.

[57] A. E. Chavarria, C. Galbiati, X. Li, and J. A. Rowlands (2016), 1609.03887.

[58] K. Abe et al. (Hyper-Kamiokande Proto-Collaboration), PTEP 2015, 053 C02 (2015), 1502.05199.

[59] R. Acciarri et al. (DUNE) (2015), 1512.06148.

[60] F. An et al. (JUNO), J. Phys. G43(3), 030401 (2016), 1507 . 05613.

[61] M. G. Aartsen et al. (IceCube) (2016), 1607.02671.

[62] S. Adrian-Martinez et al. (KM3Net), J. Phys. G43(8), 084001 (2016), 1601.07459. 